Illinois State University

ISU ReD: Research and eData

Theses and Dissertations

3-31-2019

\title{
Effects Of Attentional Focus Cues On Soccer Trap Kinematics And Performance
}

Ladule Lako LoSarah

Illinois State University, ladule.lako.losarah@gmail.com

Follow this and additional works at: https://ir.library.illinoisstate.edu/etd

Part of the Biomechanics Commons

\section{Recommended Citation}

Lako LoSarah, Ladule, "Effects Of Attentional Focus Cues On Soccer Trap Kinematics And Performance" (2019). Theses and Dissertations. 1110.

https://ir.library.illinoisstate.edu/etd/1110

This Thesis is brought to you for free and open access by ISU ReD: Research and eData. It has been accepted for inclusion in Theses and Dissertations by an authorized administrator of ISU ReD: Research and eData. For more information, please contact ISUReD@ilstu.edu. 


\section{EFFECTS OF ATTENTIONAL FOCUS CUES ON SOCCER TRAP KINEMATICS AND PERFORMANCE}

\section{LADULÉ LAKO LOSARAH}

\section{Pages}

The purpose of this research was to assess the kinematic and performance differences of an inside of the foot soccer ball control movement in response to internal, external, or holistic attentional focus coaching cues. The researchers used a pendulum catapult to launch a soccer ball from 8.5 meters at the participant. Ten female active NCAA division 1 soccer players received one of three cues, or no cue, and then had five trials to control the ball onto an artificial turf mat to score 1-3 points depending on where the ball landed. Participants completed a manipulation check survey to ascertain the efficacy of the coaching cues. A motion capture system was used to quantify the movement and processed through Visual3D software for the joint angles during 10 frames before and after ball contact. A repeated-measures ANOVA found no significant difference in any of the kinematic variables nor the performance measures between the different cuing conditions; however, a multivariate ANOVA uncovered a significant difference in performance data indicating a higher performance in the holistic cue (HC) condition $(0.68 \pm .40)$ compared to no cue (NC) condition ( $0.28 \pm 0.25)$. The means of the survey questions indicated strong compliance with the instructions. The results indicated that single word cuing may facilitate optimal performance and the high variability may support Bernstein’s analysis (1967). Further analysis is required to assess different kinematic variables and time intervals within the movement. 
KEYWORDS: attentional focus; soccer kinematics; motor control; coaching cues; soccer trap 


\title{
EFFECTS OF ATTENTIONAL FOCUS CUES ON SOCCER TRAP KINEMATICS AND PERFORMANCE
}

\author{
LADULE LAKO LOSARAH
}

\begin{abstract}
A Thesis Submitted in Partial Fulfillment of the Requirements for the Degree of

MASTER OF SCIENCE
\end{abstract}

School of Kinesiology and Recreation

\section{ILLINOIS STATE UNIVERSITY}




\section{Anti-Copyright 2019 Ladule Lako LoSarah}

This document can be accessed or used in whole or in part by any person for any reason with or without attribution to the author. Knowledge belongs to all of humanity. 


\section{EFFECTS OF ATTENTIONAL FOCUS CUES ON SOCCER TRAP KINEMATICS AND PERFORMANCE}

\section{LADULE LAKO LOSARAH}

COMMITTEE MEMBERS:

Adam E. Jagodinsky, Chair

Michael Torry

Peter J. Smith 


\section{ACKNOWLEDGMENTS}

Without the contributions, guidance, and support of many, this project would have remained a fleeting idea, rather than becoming a concrete capstone study. My sincere gratitude goes to Dr. Jagodinsky for his guidance, support, and effort in helping me overcome every challenge faced in the process of researching and writing this project. Additionally, I would to thank Dr. Torry and Dr. Smith for their contributions and feedback which turned this idea into a reality. My thanks also go to my peers who volunteered hours of their own time to help with data collection and processing, and particularly Sean Higinbotham for being the biomechanics lab wizard, slaying every technical hurdle I faced with patience and skill. Finally, I give thanks to the participants in the study and the coaching staff from the ISU women's soccer program who made this project possible. 


\section{CONTENTS}

Page

ACKNOWLEDGMENTS

TABLES

FIGURES

CHAPTER I: EFFECTS OF ATTENTIONAL FOCUS CUES ON SOCCER TRAP

KINEMATICS AND PERFORMANCE 1

$\begin{array}{ll}\text { Introduction } & 1\end{array}$

Attentional Focus 1

Soccer Biomechanics 3

Synthesis of Attentional Focus and Soccer Biomechanics for Soccer Trap

$\begin{array}{ll}\text { Analysis } & 4\end{array}$

$\begin{array}{ll}\text { Methods } & 4\end{array}$

$\begin{array}{ll}\text { Research Design } & 4\end{array}$

$\begin{array}{ll}\text { Participants } & 5\end{array}$

$\begin{array}{ll}\text { Apparatus and Task } & 5\end{array}$

$\begin{array}{ll}\text { Data Collection } & 8\end{array}$

$\begin{array}{ll}\text { Data Processing } & 10\end{array}$

$\begin{array}{ll}\text { Data Analysis } & 10\end{array}$

$\begin{array}{ll}\text { Results } & 11\end{array}$

$\begin{array}{ll}\text { Discussion } & 12\end{array}$

$\begin{array}{ll}\text { REFERENCES } & 17\end{array}$ 
APPENDIX A: RECRUITMENT SCRIPT, DATA COLLECTION SCRIPT, POST-

COLLECTION SURVEY 


\section{TABLES}

Table

Page

1. Kinematic (degrees) and performance (points) variables mean with standard

deviation in the parentheses. EC=External Cue, HC=Holistic Cue, IC=Internal Cue, NC=No Cue

2. Manipulation check survey statements given at the end of the protocol

3. Responses to survey statements with mean score across all participants and standard deviation in the parentheses 


\section{FIGURES}

Figure $\quad$ Page

$\begin{array}{ll}\text { 1. The ball serving pendulum } & 6\end{array}$

2. The scoring mat (4’x6') with point zones, 3 is closest to subject 7

3. An example of the movement the task is attempting to replicate (Online Soccer

$\begin{array}{lr}\text { Academy) } & 8\end{array}$

4. A subject performing a trial during data collection 8

5. Retroreflective marker placement on the subject 9 


\section{CHAPTER I: EFFECTS OF ATTENTIONAL FOCUS CUES ON SOCCER TRAP}

\section{KINEMATICS AND PERFORMANCE}

\section{Introduction}

Central to effective coaching is facilitating motor skill acquisition and facilitating peak performance in players. Verbal instructions are an important means by which coaches are able to guide performers to the most effective and efficient movement patterns to achieve a desired outcome.

\section{Attentional Focus}

Research has indicated that the focus of attention which verbal instructions induce, either internal or external, have an "important impact on the effectiveness and efficiency of motor performance” (Wulf, 2013). An internal focus of attention directs the performer's conscious thought toward their body movements and with verbal cues that explicitly reference one or more of their body parts (Wulf, et al., 2001). Contrarily, an external focus of attention directs the performer's conscious thought toward the effect or outcome of their movement, with verbal cues that reference implements or apparatuses as well as outcome goals (Wulf, et al., 2001). Dimmock and Gucciardi (2008) have proposed a third attentional focus paradigm, holistic focus, which consists of single word verbal cue that metaphorically summarizes the desired quality of the movement outcome without referring to outcome nor coordination patterns. Previous research has indicated that external and holistic cues enable higher performance outcomes and movement efficiency.

The theoretical basis for this observed effect is Prinz’s (1997) common-coding theory of perception and action along with the constrained-action hypothesis (Wulf, 2001). Adding to this 
understanding, Russell (2007) explained this effect within the framework of Bernstein’s (1967) synergy theory.

Dimmock and Gucciardi (2008) revealed increased performance during golf putting when participants employed a single holistic word cue. Furthermore, Wulf et al. (1999) asked novice participants during a golf chip shot to either focus on their arm swing (internal focus) or the club swing (external focus). The results indicated that the group with the external focused cue, club swing, scored significantly higher than the group who received the internal focused cue, arm swing. Additionally, qualitative analyses have been conducted as Wulf et al. (2002) had experienced volleyball coaches observe the serve of novice and advanced players who had received either an internal focus or external focus cue. The coaches evaluated the movement and scored the external focus group higher than the internal focus group. For muscle activation, Marchant et al. (2008) uncovered a decrease in EMG activity paired with increased peak joint torque when a bicep curl was performed with an external focus of attention. At present, however, kinematic analysis of movement performance under different induced attentional focus conditions is conspicuously absent within the literature.

One of the only studies to connect attentional focus and complex movement kinematics was a 2009 investigation of novice jugglers. Zentgraf and Munzert (2009) measured the upper body kinematics and ball trajectory of three groups of learning jugglers: one who received ballrelated cues; one who received body-related cues; and a no cue group. They uncovered that while all three groups improved in a retention test, there were significant differences in upper body kinematics and ball trajectories during the juggling task. There was a lack of significant differences between the no cue group and the external cue group. This study focused on learners and examining the effects on expert performers was beyond the scope of the investigation. In 
another similar study, Lohse et al. (2010) found increased shoulder kinematic variability under external cue conditions for dart throwing experts. These kinematic effects of attentional focus cues have yet to be assessed in regards to a soccer trap among expert performers.

\section{Soccer Biomechanics}

Purported as the "defining action of soccer," the biomechanical analyses of soccer techniques have focused primarily on the kick (Lees et al., 2010). Numerous previous studies have assessed, quantified, and analyzed a wide variety of common soccer kicking techniques from the standpoint of kinematics, kinetics, EMG, and motor control (ibid.). Perhaps equally as important as kicking is the technique of controlling or "trapping” a soccer ball. Tahara et al. (2012) posit that the inside of the foot soccer trap may in fact be the most frequently employed skill during a soccer match.

Despite the significance of this technique, little biomechanical research has examined trapping in soccer. In one previous study, Asai et al. (1981) suspended a soccer ball from the ceiling and swung it pendulum-like toward a subject, quantifying the movement with an accelerometer and cinematography. Given the unrealistic nature of the ball delivery method compared to an in-game situation, this study's relevance is its pioneering inquiry into the analysis of soccer trapping with a cinematographic technique and accelerometer.

The only other biomechanical analysis of soccer trapping, Tahara et al. (2012), engaged 13 experienced male collegiate soccer players and kicked a ball for them to trap from 7 meters away at random speeds. Measurement was conducted via triaxial accelerometers under the lateral malleolus and on the lateral side of the fifth metatarsal bone, along with two high speed video cameras recording at $200 \mathrm{~Hz}$. The researchers analyzed 10 frames before and 10 frames after ball 
contact, finding a significant relationship between the external rotation of the hip and knee with ball deceleration at ball contact. These findings guided the present study’s analysis.

\section{Synthesis of Attentional Focus and Soccer Biomechanics for Soccer Trap Analysis}

Biomechanical analytics provide a valuable entry into the understanding of motor control theory. Davids et al. (2000) argue that an incorporation of the two sub-disciplines is required to "understand how organization of the movement system changes during performance and development.” The implications for effective soccer coaching are evident, and it is within this scope that the present study aims to integrate motor control with biomechanical analysis: to examine how soccer trapping performance and kinematic movement is affected by different coaching cues. The hypotheses of this study are: 1.there will be higher performance scores during the external and holistic cue conditions than the no cue and internal cue conditions; 2. there will be underlying kinematic differences that accompany the performance differences across conditions.

\section{Methods}

\section{Research Design}

The study was a single factor design consisting of one within subjects factor (cue type) with four levels. The dependent variables included 10 kinematic variables: knee flexion at ball contact (KX_BC); hip flexion at ball contact (HX_BC); hip rotation displacement 10 frames before ball contact (HZ_DIS_PRE); the average hip rotation position over the 10 frames before ball contact (HZ_AVG_PRE); hip rotation displacement 10 frames after ball contact (HZ_DIS_POST); the average hip rotation position over the 10 frames after ball contact (HZ_AVG_POST); knee rotation displacement 10 frames before ball contact (KZ_DIS_PRE);

the average knee rotation position over the 10 frames before ball contact (KZ_AVG_PRE); knee 
rotation displacement 10 frames after ball contact (KZ_DIS_POST); the average knee rotation position over the 10 frames after ball contact (KZ_AVG_POST); and score.

\section{Participants}

Following approval by the institutional review board, 10 current female NCAA Division I soccer players from the ISU team consented to participate in this study (Age: $19.4 \pm 0.97$ years, Height: $1.65 \pm 0.05 \mathrm{~m}$, Weight: $64.27 \pm 6.88 \mathrm{~kg}$ ). The players were currently in season, physically cleared by a trainer and physician to participate in intercollegiate athletics, free from injury for the previous year, and had a minimum of 12 years of competitive soccer experience (M: 14.8 \pm 2.1 years). All were right foot dominant players, except for one, who was left foot dominant. For their primary positions, four were midfielders, four were defenders, and two were forwards.

\section{Apparatus and Task}

A custom pendulum was designed to kick a standard size 5 soccer ball (Adidas, Germany) at the participant from a distance of 8 meters at a speed averaging $14.5 \mathrm{~m} / \mathrm{s}$ (see figure 1). The ball arrived at the knee height of each participant and had to arrive within a 2 foot “strike-zone," marked with blue tape (see figure 2). 


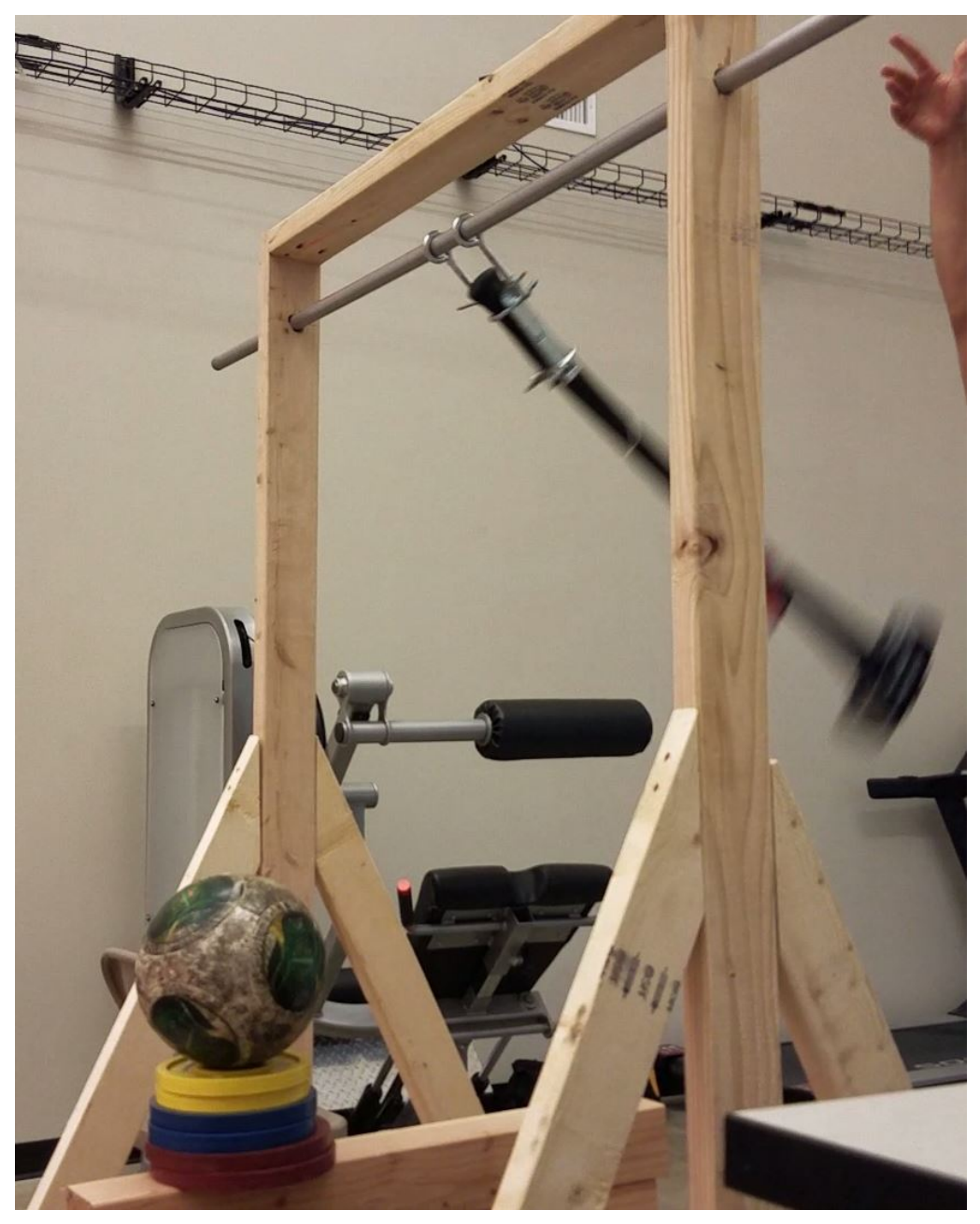

Figure 1. The ball serving pendulum.

The participant had to execute an inside of the foot trap (see figure 3) with their dominant foot to control the soccer ball on a 6'x4' turf mat divided into three scoring zones with 3 points being the closest 2 feet of the mat, 2 points within the middle 2 feet, and 1 point for stopping the 
ball in the last 2 foot zone (see figure 2). Any ball that stopped off of the mat earned zero points.

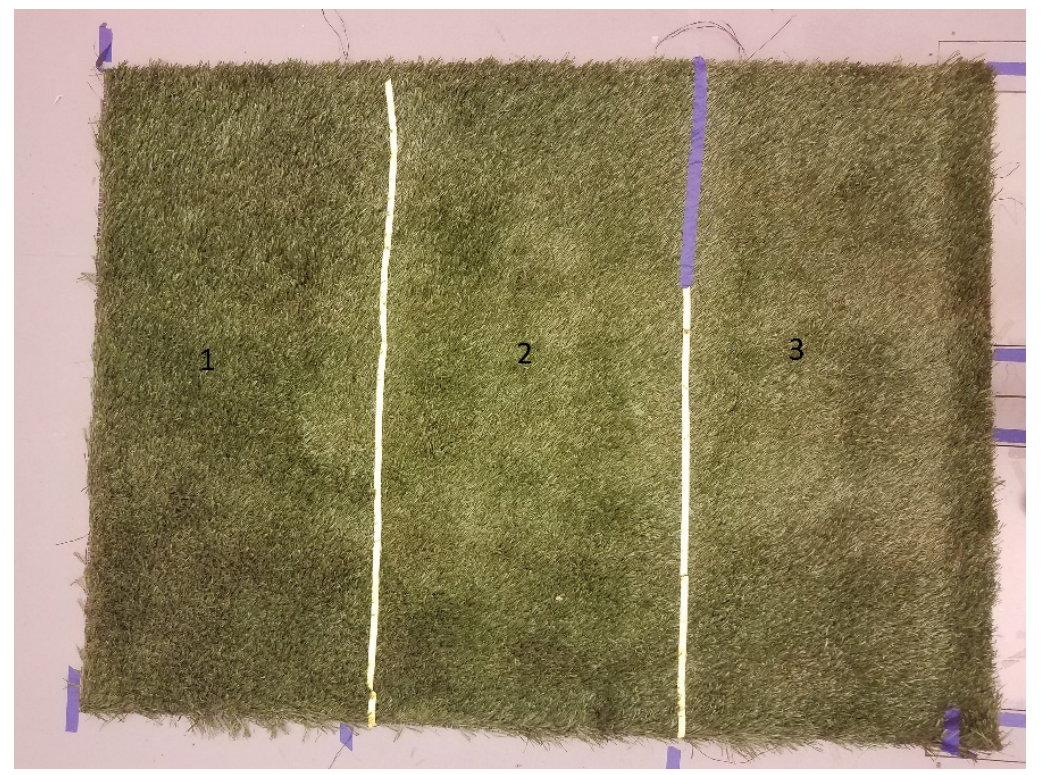

Figure 2. The scoring mat (4'x6') with point zones, 3 is closest to subject.

There were four conditions: No Cue, the participant was told to simply score the highest amount of points; Internal Cue (IC), the participant was told "to move their foot back when their foot makes contact with the ball;" Holistic Cue (HC), the word "cushion” was told to the participant before each trial; and External Cue (EC), the participant was told to "meet and guide the ball into the scoring zone.” These cues were adapted from an online video teaching the technique of the inside of the foot soccer trap (Online Soccer Academy, 2013). 


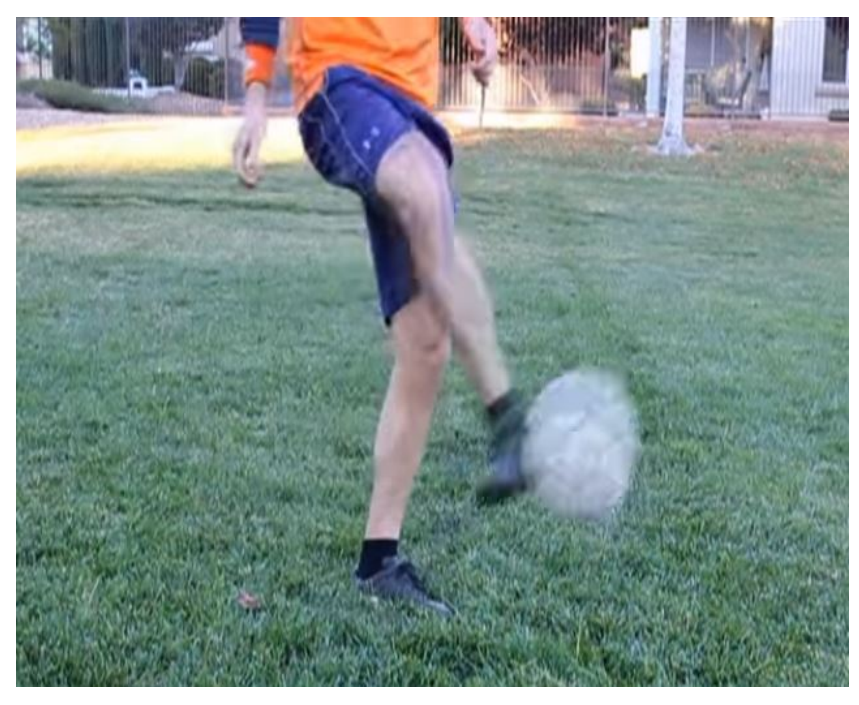

Figure 3. An example of the movement the task is attempting to replicate (Online Soccer Academy)

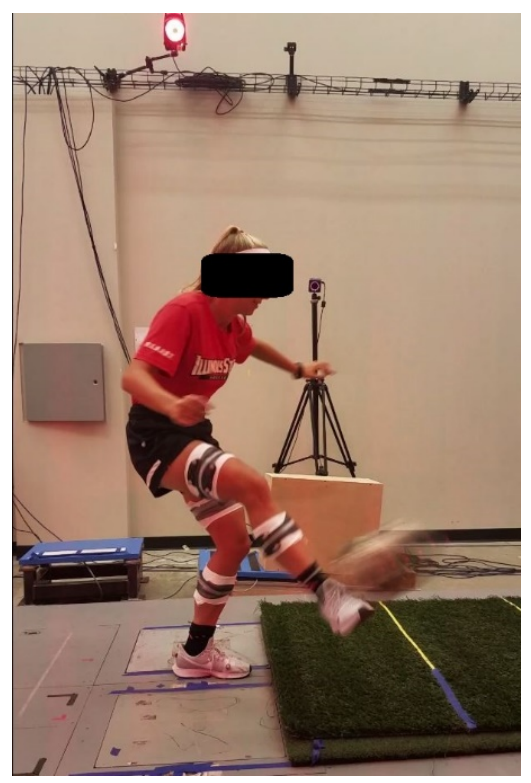

Figure 4. A subject performing a trial during data collection.

\section{Data Collection}

Thirty-three 14mm retroreflective markers (MKR-6.4, B\&L Engineering, Tustin, California, USA) were attached to the participant bilaterally at the anatomical landmarks of 1st metatarsal, 5th metatarsal, heel, medial and lateral malleolus, medial and lateral knee, greater trochanters, iliac crests, and sacrum. For the static calibration capture all markers were present, 
after which markers on the ankles and knees were removed for the dynamic movements.

Additionally, two tape markers were attached to the ball to record the position, velocity, and acceleration of the ball.

Participants performed a soccer specific warm up as they would before a game or training session, after which they proceeded to execute 10 familiarization trials of the task. After they were warmed up and familiar with the task, the participants executed five "no cue” recorded trials. After the "no cue” trials, the subsequent cue conditions were randomly assigned, and each participant executed 5 trials of each condition.

At the end of the entire protocol, the participants answered a five question survey which served to assess the cues' manipulation level. The survey had a scale of 1-5 with 1 meaning "strong disagreement" with the statement and 5 meaning "strong agreement" with the statement (Becker and Smith, 2013).

Measurement of segment motion was obtained using a 16-camera Vicon MX optical motion capture system (Vicon® ${ }^{\circledR}$, Los Angeles, CA, USA) with a sampling frequency of $200 \mathrm{~Hz}$. For each trial, data from 10 frames before ball contact to 10 frames after ball contact was collected for analysis.

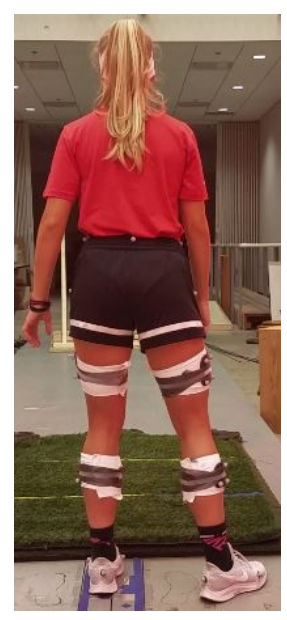

Figure 5. Retroreflective marker placement on the subject. 


\section{Data Processing}

Marker trajectories were lowpass filtered $(6 \mathrm{~Hz})$ and local segment axes were established from global laboratory coordinates using an X-Y-Z Cartesian rotation sequence. Visual 3D software (C-Motion Research Biomechanics, Germantown, Maryland, USA) was used to calculate relative segment angles. The movement was broken down into three phases: Phase 1. 10 frames before ball contact; Phase 2. ball contact; and Phase 3. 10 frames after ball contact. The frame of ball contact was inferred from the acceleration of the ball, precisely the moment where the graph of ball acceleration changed from negative (moving towards the participant) to positive (moving away from the participant). A MATLAB script then pulled and aggregated the data from the relevant frames. Pre- and post-ball contact movement variables were broken down into two discrete variables, displacement: the final frame minus the first frame to capture the movement of the segment; and average position: the average of segment angle over the 10 frames.

\section{Data Analysis}

All variables were tested for normality via Mauchly’s test of sphericity and confirmed a failure to reject of the null hypothesis of equal variance with a Greenhouse-Geisser $p$-value of greater than 0.05 for every variable. A repeated-measures ANOVA as well as a multivariate ANOVA were then used to detect differences in the means of the kinematic and performance variables between the different conditions. A p-value of less than 0.05 was regarded as a statistically significant difference. Statistical analyses were carried out using IBM SPSS Statistics v22.0.

The survey data were averaged across participants with standard deviation calculated. 


\section{Results}

A repeated measures ANOVA revealed a lack of significant differences between the different conditions for all kinematic and performance variables ( $p>0.05)$. A multivariate ANOVA uncovered a significant difference for the Score variable, with a pairwise post-hoc test indicating a highly significant difference between the Holistic Cue (HC) and No Cue (NC) conditions $(p<.01)$.

Table 1. Kinematic (degrees) and performance (points) variables mean with standard deviation in the parentheses. EC=External Cue, HC=Holistic Cue, IC=Internal Cue, NC=No Cue.

\begin{tabular}{|l|l|l|l|l|}
\hline Variable & EC & HC & IC & NC \\
\hline Score & $0.54(.40)$ & $0.68(.40)$ & $0.64(.44)$ & $0.28(.25)$ \\
\hline KX_BC & $-73.43(16.37)$ & $-70.02(16.11)$ & $-73.66(19.10)$ & $-74.11(19.00)$ \\
\hline HX_BC & $87.59(15.06)$ & $88.72(16.53)$ & $88.57(14.70)$ & $88.63(13.86)$ \\
\hline HZ_DIS_PRE & $-3.25(2.01)$ & $-2.87(2.22)$ & $-4.07(3.19)$ & $-3.16(2.06)$ \\
\hline HZ_AVG_PRE & $-16.21(13.66)$ & $-12.71(17.62)$ & $-15.70(14.22)$ & $-12.67(19.62)$ \\
\hline HZ_DIS_POST & $3.34(4.20)$ & $2.20(4.08)$ & $2.73(3.77)$ & $2.99(4.07)$ \\
\hline HZ_AVG_POST & $-16.72(12.11)$ & $-13.65(16.31)$ & $-17.29(12.63)$ & $-13.55(17.29)$ \\
\hline KZ_DIS_PRE & $-1.59(1.91)$ & $-1.25(2.40)$ & $-0.90(2.41)$ & $-1.99(2.07)$ \\
\hline KZ_AVG_PRE & $-12.53(10.88)$ & $-8.50(13.84)$ & $-13.28(9.64)$ & $-6.42(13.77)$ \\
\hline KZ_DIS_POST & $3.34(4.20)$ & $2.20(4.08)$ & $2.73](3.77)$ & $2.99(4.07)$ \\
\hline KZ_AVG_POST & $-13.65(10.64)$ & $-9.69(14.38)$ & $-14.31(9.90)$ & $-8.35(15.26)$ \\
\hline
\end{tabular}

Table 2. Manipulation check survey statements given at the end of the protocol.

\begin{tabular}{|l|}
\hline Statement \\
\hline 1. When the cue mentioned my foot, I focused on my foot while performing the trap. \\
\hline 2. When the cue mentioned the ball, I focused on the ball while performing the trap. \\
\hline 3. When the cue mentioned neither the ball nor my foot, I focused on the whole movement. \\
\hline 4. I simply performed a trap as I normally would regardless of which cue was said to me. \\
\hline 5. I focused on the instructor's cues while performing the trap. \\
\hline
\end{tabular}


Table 3. Responses to survey statements with mean score across all participants and standard deviation in the parentheses.

\begin{tabular}{|l|l|l|l|l|}
\hline S1 & S2 & S3 & S4 & S5 \\
\hline 4.6 & 3.9 & 4.5 & 2 & 4.9 \\
$(0.52)$ & $(0.88)$ & $(0.71)$ & $(0.94)$ & $(0.32)$ \\
\hline
\end{tabular}

\section{Discussion}

The aim of the study was to examine the effects of different attentional focus cues on the performance and kinematics of an inside of the foot soccer trap. The results showed that although there was an absence of difference in kinematics across the conditions, there was an improvement in performance during the Holistic Cue condition compared to the No Cue condition, as hypothesized. These findings show that single-word Holistic Cues may be employed by coaches to facilitate optimal performance in expert soccer players during the execution of common soccer techniques. Furthermore, these findings confirm Gucciardi and Dimmock’s (2008) assertion that holistic cue words reduce anxiety and improve performance among expert performers.

A theoretical explanation for this effect could be that focusing on a global movement cue prevents the performer from concentrating on one segment of the movement and consequently disrupting the natural synergy of muscles (Russell, 2007). Russel explains further that an effort to constrain any one part of a complex motor system can have unintended influences on other segments. Support for this assertion is further corroborated by the results of Vance et al. (2004) and Marchant et al. (2008)which showed interfering co-contraction of the antagonist muscles via EMG activity during a bicep curl with an internal focus of attention. Additionally, Wulf and Dufek (2009) uncovered increased joint torques during a vertical jump with an external focus of attention versus an internal focus of attention. These results indicate more efficient synergistic 
muscle actions during an external or holistic cue attentional focus compared to no cue or internal cue conditions.

Further support for the Bernstein synergistic model of complex system movement is the high variability in kinematic patterns with the relative consistency of performance scores, which lacked significant difference across all but the No Cue compared to the Holistic Cue conditions. Bernstein (1967) observed that the outcomes of movements are relatively invariant despite high variation within joint movements from repetition to repetition. With a complex movement that has many degrees of freedom, there are various optimal movement solutions to achieve the movement outcome goal. He exemplified this is demonstrating that the trajectories of hammer swings among expert blacksmiths were consistent despite wide variations in shoulder joint movement kinematics (Russel, 2007). Among experts, the endpoint can be achieved via various movement patterns. This is additionally further support for the choice to focus on the defining moment of the trap—ball contact—as well as the directly preceding and ensuing movements.

This theoretical explanation is subtly yet distinctly divergent from Wulf's (2001) constrained action hypothesis or Prinz's (1997) common-coding theory in that rather than define the approach to movement as unconscious or conscious control of the performer's own body segments, it highlights the importance of the variable of control to the movement outcome (Russell, 2007).

An inquiry into the coaching cues and manipulation demonstrates a strong compliance by the participants as well as cues being in line with Wulf's (2002) and Dimmock and Gucciardi's (2008) criteria for internal, external, and holistic focus-inducing verbal instructions. When asked if they "simply performed the trap as they normally would," all except for two subjects strongly disagreed. Furthermore, all subjects reported focusing on their foot when the cue mentioned their 
foot, focusing on the ball when the cue mentioned the ball, and focusing on the complete movement when the cue mentioned neither the ball nor their foot. At the level of the verbal cue, Wulf (2013) insists that the internal and external instructions must express the same idea in relatively the same language except that the internal cue must mention a body part while the external cue instructions avoid referencing a body part. In the present study, both sets of instructions included the idea of having the subject move their foot towards the ball before contact, then move their foot back after contact, referencing only the foot in the internal cue instructions, while referencing only the ball in the external cue instructions. For the holistic cue, in accordance with Dimmock and Gucciardi (2008), the instructions consisted of a single word that summarized the entre movement: “cushion.” In respect to these experimental assessments, the instructions and manipulation were successful and validated by the existing literature. Nonetheless, an improvement could have been to administer the survey after each condition, rather than at the end of all trials, to allow for a statistical analysis of differences in survey responses between conditions.

A success of the present study's kinematic analysis is the confirmation of the accuracy of the soccer ball delivery method via the pendulum catapult. The lack of significant differences of sagittal plane kinematics for the hip and knee at ball contact indicate that the ball was arriving to the subject in a relatively consistent position during each trial. This provides a substantial improvement to the methodology employed by Tahara et al. (2012), who had a ball kicked at random speeds for a trap, and Asai et al. (1981), who unrealistically swung a suspended ball into the subject for recording a trap. The ball delivery method of the present study allows for further study of this particular movement to be reliable and realistic. Given this platform, further 
analysis must be conducted to better understand the soccer trap movement as a whole, and then determine where kinematic differences related to performance could occur during the movement.

Although the task was a replication of a commonly executed skill in soccer, the laboratory setting, as well as the layout of the scoring mat and the apparatus serving the ball rather than another player, could have disrupted the subject's technique. Since the subjects were standing on floor with the raised turf mat placed about 6 inches in front of them, it could have forced them to feel uncomfortable and fail to perform the movement as they would when standing on the surface where they have to control the ball. In this way, a design improvement would have employed a larger turf mat that allowed the participant to be able to move more freely and control the ball in a less restrained manner.

The no cue performance scores were the lowest on average across all conditions. This could be due to the fact that the no cue condition always occurred first, to ensure that it was absolutely free of manipulation, and therefore the subjects were not yet comfortable with the task. However, since the movement is a comment technique among expert soccer players and the subjects completed a set of familiarization trials, unease with the movement is a potential but inconclusive explanation for the low no cue condition scores. The randomized order may have failed to control for order effects given the small sample size.

Although the examination for kinematic differences between conditions proved to be inconclusive, there could be methodological and technical limitations which caused this finding. Primarily, 10 frames before and after ball contact, representing 1/20 of a second, is too short of a time to assess the subtle movements occurring during the soccer trap. A further analysis should examine a larger interval of the movement. Movement timing along with muscle synergies via EMG could additionally uncover relevant findings. Secondly, the kinematic variables, the 
particular joints, and their movement in the specific planes could have been insufficient for the detection of movement pattern disruption due to coaching cues. Initial analysis and quantification of the soccer trap movement must precede a venture into how coaching cues affect the movement.

The search of practical applications to the field of soccer coaching was the explicit intention of the present study_-particularly in the question of how do a coach's verbal cues influence movement patterns during the execution common soccer techniques in respect to common-coding theory, the constrained-action hypothesis, and complex system synergies. The findings conclude that adopting holistic coaching cues may lead to higher performance measures in expert soccer players while executing common soccer-specific techniques. Additionally, there is support that complex system theory could explain this effect rather than common-coding theory or the constrained-action hypothesis. Although the present analysis proved inconclusive on the kinematic level, the study establishes a useful and novel framework through which further analyses may potentially uncover significant results. 


\section{REFERENCES}

Asai, T., Kobayashi, K. \& Matsumoto, M. (1981). A biomechanical study of ball stopping skills in soccer. Japanese Society of Physical Education, 26(3), 245-251.

Becker, K., \& Smith, P. J. K. (2013). Age, Task Complexity, and Sex as Potential Moderators of Attentional Focus Effects. Perceptual and Motor Skills, 117(1), 130144. https://doi.org/10.2466/23.25.PMS.117x14z3

Bernstein, N. A. (1967). The co-ordination and regulation of movements. Oxford: Pergamon Press.

Davids, K. , A. Lees \& L. Burwitz (2000) Understanding and measuring coordination and control in kicking skills in soccer: Implications for talent identification and skill acquisition, Journal of Sports Sciences, 18:9, 703-714, DOI:

\section{$10.1080 / 02640410050120087$}

Gucciardi, Daniel, James A. Dimmock (2008), Choking under pressure in sensorimotor skills: Conscious processing or depleted attentional resources?, Psychology of Sport and Exercise, Volume 9, Issue 1, Pages 45-59

Handford, C., Davids, K., Bennett, S. and Button, C. (1997). Skill acquisition in sport: Some applications of an evolving practice ecology. Journal of Sports Sciences, 15, 621- 640.

Lees A, Asai T, Andersen TB, Nunome H, Sterzing T. The biomechanics of kicking in soccer: a review. J Sports Sci. 2010 Jun;28(8):805-17. Doi: 10.1080/02640414.2010.481305. Review. PubMed PMID: 20509089.

Lohse, K. R., Sherwood, D. E., \& Healy, A. F. (2010). How changing the focus of attention affects performance, kinematics, and electromyography in dart throwing. Human Movement Science, 29(4), 542-555. https://doi.org/10.1016/j.humov.2010.05.001 
Russell, D. Attentional Focus on the Invariant Control Variables. E-Journal Bewegung und Training.1 (2007), 47-48

Schmidt, R. A. (1999) Motor control and learning. A behavioral emphasis, 3nd ed. Human Kinetics

Tahara, R., Shimonagata, S., \& Taguchi, M. (2012). (2012). Relationship between acceleration of the foot and lower-leg movement in soccer ball trapping. Paper presented at the 30th Annual Conference of Bimechanics in Sports, 303-306.

Vance J, Wulf G, Töllner T, McNevin N, Mercer J. EMG activity as a function of the performer's focus of attention. J Mot Behav. 2004 Dec;36(4):450-9. PubMed PMID: 15695233.

Williams, A.M., Davids, K. and Williams, J.G. (1999). Visual Perception and Action in Sport. London: Routledge.

Wulf G, McConnel N, Gärtner M, Schwarz A. Enhancing the learning of sport skills through external-focus feedback. J Mot Behav. 2002 Jun;34(2):171-82.PubMed PMID: 12057890.

Wulf G (2013): Attentional focus and motor learning: a review of 15 years, International Review of Sport and Exercise Psychology, 6:1, 77-104

Wulf, G., Ho“ß, M., \& Prinz, W. (1998). Instructions for motor learning: Differential effects of internal versus external focus of attention. Journal of Motor Behavior, 30, 169-179 


\section{APPENDIX A: RECRUITMENT SCRIPT, DATA COLLECTION SCRIPT, POST-}

\section{COLLECTION SURVEY}

\section{RECRUITMENT SCRIPT}

I would like to invite you to participate in a research study to understand the effects of different verbal cues on motor control. You are eligible to participate if you are 18 years of age or older, have not had a musculoskeletal disorder/injury in the legs and/or feet or low-back pain within the past year, and are not currently pregnant. If you agree to participate, you will be asked to report to the ISU Biomechanics Laboratory (MCH 185C) for approximately 40-60 min for data collection. Participants will wear athletic compression clothing during the data collection to avoid disruption of equipment to be placed on the clothing. Prior to data collection, reflective markers will then be placed on participant's pelvis and legs to allow motion capture. Each participant will be assigned a group and perform a soccer trap after receiving a specific soccer cue. The ball will be rolled down a ramp and you will be asked to control it with the inside of your foot as you would in a match. The group to which each participant belongs will be randomly assigned. The results of the research may be published, but your name will not be used. If you choose not to participate or wish to withdraw from the study at any time during the study, there will be no penalty to you.

Potential risks to the participants are minimal, but may include fatigue, aching or pain in the lower legs due to prolonged standing. There are no direct benefits to participating in this study.

If you would like to participate in this research, or if you have questions later, please contact Dr. Adam Jagodinsky at aejagod@ilstu.edu or Ladule L. LoSarah at llakolo@ilstu.edu 


\section{SOCCER TRAP PROTOCOL SCRIPT}

-We will be investigating how different coaching cues affect your performance of an inside of the foot ball control.

-Your task will be to control a soccer ball launched from 12 yards away with the inside of your foot into a marked scoring zone on the turf mat. You will get a score for each ball.

-After having reflective markers placed on your lower body, you will do a soccer specific warmup, as you would before a game or practice, and then complete 3 trials to get the hang of the activity, followed by 20 recorded trials, split into groups of 5 with a 2 minute break in between. -Your goal is to achieve the highest score possible. In the first zone, a score of 3 points will be awarded, in the $2^{\text {nd }}$ zone, 2 points, and in the far zone, 1 point. If the ball does not stay on the turf mat, 0 points will be awarded for that trial.

**Familiarization trial**

-Now we will collect scored trials.

${ }^{* *}$ Complete 5 scored trials**

-Now we will take a 2 minute break.

-Now I will give you a coaching cue before each trial and I would like you to focus on executing the trap in reference to the cue given.

Random order:

INT CUE: Move your foot forward to meet the ball and move your foot back on contact

**Cue before each trial, collect 5 trials**

-Now we will take a 2 minute break.

EXT CUE: Meet the ball and guide the ball

**Cue before each trial, collect 5 trials** 
-Now we will take a 2 minute break.

HOL CUE: Cushion

**Cue before each trial, collect 5 trials**

-We have finished collecting data, please complete a short survey about the data collection process. Thank you for your time and effort.

\section{POST-PROTOCOL MANIPULATION CHECK SURVEY}

Please circle the number on a scale from 1-5 (1 being the least, 5 being the most) how much you agree with each of the following statements:

1. When the cue mentioned my foot, I focused on my foot while performing the trap.

1 2 3 4 5

2. When the cue mentioned the ball, I focused on the ball while performing the trap.

$\begin{array}{lllll}1 & 3 & 4 & 5\end{array}$

3. When the cue mentioned neither the ball nor my foot, I focused on the whole movement.
1
2
3
4
5

4. I simply performed a trap as I normally would regardless of which cue was said to me.

$\begin{array}{lllll}1 & 2 & 3 & 4 & 5\end{array}$

5. I focused on the instructor's cues while performing the trap.

1 